\title{
LA FÓRMULA DEL CUERPO SIN ÓRGANOS UNA APROXIMACIÓN BERGSONIANA A SU ENUNCIACIÓN
}

Miguel Ruiz Stull*

RESUMEN: Tomada desde Artaud por Deleuze ya desde la redacción de Lógica del sentido (1969), la expresión de cuerpo sin órganos ( $\mathrm{CsO}$ ) no deja de causar al menos perplejidad. En su enunciación se traman puntos cruciales de la filosofía de Deleuze desde su teoría del acontecimiento y de la diferencia, pasando por una definición y una analítica del deseo, hasta una determinada noción de vida que articularía el proceso de su generación. Sin desestimar lo anterior y los profusos usos y determinaciones actuales que ha cobrado esta noción, nuestro propósito es vincular al CsO con la concepción de vida que según nuestra lectura, se halla en la base de su formulación. Esto permanece en el texto como subyacente, y nuestra propuesta se inscribe en determinar el grado de influjo que posee el vitalismo de Bergson en la concepción de esta fórmula: desde este punto de vista es plausible sostener que el $\mathrm{CsO}$ es una operación que se funda de modo crucial en una lectura atenta al élan vital de bergsoniano. En suma, si el CsO es una involución creadora por definición de su proceso, no es sino porque su puesta en marcha significa y se efectúa en una constante y radical lucha que implica cada vez el desmontaje o desmembramiento de la idea de organismo.

PALABRAS CLAVES: G Deleuze. H Bergson. Vida. Organismo. CsO

In nova fert animus mutatas dicere formas corpora. di, coeptis - nam vos mutastis et illas adspirate meis primaque ab origine mundi ad mea perpetuum deducite tempora carmen

Ovidius

\footnotetext{
• Universidad de Chile y Universidad Diego Portales. E-mail: ruizstull@gmail.com
} 
Es quizás esta, la noción de Cuerpo sin Órganos ( $\mathrm{CsO})$, una de las más sugerentes que atraviesan el pensamiento de Gilles Deleuze. Su data es antigua, su uso progresivo, en cuanto que el texto delezeano la piensa y rearticula constantemente. Su primera aparición responde a un problema planteado ya en Logique du sens (1969), en la decimotercera serie, problema que refiere esencialmente a una sugerente lectura de Artaud que vincula la práctica artística con el diagnóstico y el tratamiento psiquiátrico, en el horizonte de una teoría de la enunciación de acciones y pasiones en el marco general de la teoría estoica de los incorporales. ${ }^{1}$ No obstante, la capitalización de esta expresión se detecta en la redacción de L'Anti-Edipe (1972), trabajo que Deleuze lleva en conjunto con Guattari. Se puede decir que en esta obra la expresión del CsO se constituye como tal en una operación, proceso por medio del cual se intenta dar cuenta de los caracteres generales de la producción deseante, la cual se atreve a pensar el ser como potencia creadora sobreabundante, bajo la imagen de una energía pulsional que ya era sugerida por el pensamiento de Spinoza y Nietzsche. Desde esta perspectiva, lo que describe en su generalidad el pensamiento y operación del CsO es el planteamiento de un lugar límite de la experiencia, que con cierta justicia ha sido remitido en sus efectos directos a un problema político y ético, con todas sus posibles vinculaciones, intercambios y superposiciones. Así ha sido comprendido por Alliez (2004) en su artículo "La condición del CsO, o de la política de la sensación". Este trabajo vincula la operación del CsO a una transformación de la práctica de sí enmarcada en una propuesta ética con la construcción ethopoiética del individuo: una especie de estética de sí, fundada en una política de la sensación, en el diseño estratégico de un arte de la experimentación (Cf. ALLIEZ, 2004, p. 104). Si bien es razonable esta posición, y así lo muestra el riguroso y elocuente análisis de Alliez, queda en suspenso lo que aquí nos comienza a interesar sobre el $\mathrm{CsO}$, a saber, el fondo metafísico o la apuesta ontológica que implica la operación y el proceso que encarnaría e implicaría profundamente su práctica, formación y despliegue. Otra forma de exploración ha sido acuñada en las remisiones a las ciencias naturales, las de Wiesmann y Simondon principalmente, que vinculan la enunciación del CsO a los procesos de individuación del organismo, sustraídos de toda regla trascendente o de una apuesta funcional o finalista en los procesos involucrados en su evolución. Este punto ha sido

\footnotetext{
${ }^{1}$ Este problema tiene su más precisa presentación en este punto, que reconocemos como un eje por explorar por nuestra lectura: "si la pasión y la acción son los polos inseparables de una ambivalencia es porque los dos lenguajes que forman pertenecen inseparablemente al cuerpo, a la profundidad de los cuerpos." (DELEUZE, 1969, p. 108). El CsO, en nuestra óptica, hará extensiva esta relación biunívoca que comportaría a la naturaleza del lenguaje: a saber, la producción de un CsO debe en todo momento, como veremos, en su práctica esencial de sustraerse de estratos determinantes, y estos se configuran en cuanto su naturaleza normativa como la implementación de toda una orgánica del juicio y del discurso que afecta a una determinada organización de los cuerpos.
} 
desarrollado con extensiva precisión por Ansell Pearson en Germinal Life (1999). En ese texto se explica que el plan(o) desarrollado por Deleuze se afirma en una etología de los conjuntos. Esto se expresa en el uso que se da al plano-huevo en cuanto imagen eminente de la operación del CsO, el cual se halla librado de una continuidad dada por el germoplasma, lo cual permite pensar que "las fuerzas de la vida expresan una evolución creadora más allá de toda constricción entrópica" (p. 4). Ansell Pearson concluye que las singularidades-acontecimientos emergen a partir de la configuración de un campo de individuación que produce cada vez singularidades preindividuales, hilando así la operación del CsO con la idea de acontecimiento de Logique du sens, o bien, recordando desde Différence et répétition (1968) la afirmación de que solo lo involucionado evoluciona. ${ }^{2}$ Este punto es interesante, aunque oscuro técnicamente, ya que nos conecta con la apuesta más radical de Deleuze, que en nuestra lectura nos reenviaría directamente a la obra de Bergson, en la medida en que constantemente se pueden observar insistencias que permiten coligar uno y otro pensamiento, insistencias que si bien no son expresas literalmente, permiten, por el uso de algunas expresiones alusivas, vincularlos plausiblemente. Nuestra posición intentará dar con los caracteres esenciales de un estilo de pensamiento que pretende explicar el devenir de las formas, de los individuos, de los sujetos sobre un fondo pre-individual, pre-formal, esto es, material y fluctuante con que puede ser pensada la energía que expresan las fuerzas vitales.

\footnotetext{
$\overline{2}$ Aquí solo hallamos lo que una y otra vez el pensamiento de Deleuze rearticula; en este caso, desde Différence et répétition, reconocemos con la audaz tesis de un sujeto larvario, que deshace las categorías y determinaciones, cada vez, de un sujeto ya constituido y acabado, aquel sujeto detentado por la tradición filosófica racionalista mayor. Damos cita al contexto, que se sirve de la embriología para deshacer justamente lo estático que supone la noción funcional de organismo a la cual se opondrá la operación esencial de un CsO: "La verdad de la embriología consiste en que hay movimientos vitales sistemáticos, deslizamientos, torsiones, que solo el embrión puede soportar: el adulto quedaría destrozado. Hay movimientos de los que solo se puede ser paciente, pero lo paciente, a su vez, no puede ser más que una larva. La evolución no se hace al aire libre, y solo lo involucionado evoluciona [...] el pensamiento es más bien uno de esos movimientos terribles, que solo pueden ser soportados en las condiciones de un sujeto larvario [...] el filósofo es el sujeto larvario de su propio sistema" (DELEUZE, 1968, p. 155-156). El CsO, desde esta perspectiva, no solo replica sino que extiende esta noción de subjetividad que es propuesta en aquella obra: la cuestión, a nuestro juicio, estriba en que la realidad de un cuerpo sin órganos debe necesariamente responder a las formas tradicionales de determinación en vista de detectar aquellos puntos estratégicos donde sea posible deshacer la noción de función que permite su poder de determinación. El sujeto así, en cuanto que en vista de su constitución debe ser constituyente de las condiciones de donación de un objeto en la experiencia, ha de ser deshecho para arriesgar, tanto prudente como imprudentemente, lo veremos, sobre ese fondo cuasi trágico que atraviesa la existencia: en todos los casos se trata de la implementación de una serie de estrategias, desde las perversiones de orden psíquico, pasando por las torsiones artísticas del lenguaje, hasta el ejercicio de una resistencia sobre las formas típicas de relación social, y en este procedimiento se puede salir tanto vencedor como vencido, tanto abierto a más potencialidades como cerrado y clausurado frente a ellas; en una palabra, la cuestión que toda vez implica a un CsO es una cuestión, finalmente, de vida o muerte.
} 
Nuestro objetivo en lo que sigue será dar cuenta de las condiciones de una nueva ontología que, en nuestra hipótesis, toma por fórmula la operación del $\mathrm{CsO}$, ontología de la potencia que bien puede traducir la transformación de evolución a involución, siempre creadora de novedad y fuente de imprevisto para la experiencia en general. O bien, como ya había sido intuido, aunque no desarrollado, por Aristóteles en Física, una ontología de la potencia que da cuenta de un ser de sensación sujeto a sus propias alteraciones fundado en:

El hecho de que todas las cosas que son alteradas (alloioumenon) son alteradas (alloioustai) por los sensibles (aistheton) y que sólo hay alteración (alloiosis) en las cosas de las que se puede decir (legetai) que son esencialmente afectadas (paskhein) por la acción de los sensibles (aistheton). (245b3)

Nuestra estrategia de aproximación, sin desentenderse del todo de estas dos perspectivas ya abiertas y mencionadas, a saber, la ético-política de Alliez y la biológica de Ansell Pearson, se desplegará en dos momentos: el primero dará cuenta de los principales hitos de enunciación del CsO tal como es presentado en Mille Plateaux (1980), y desde allí vincular, en un segundo momento, estos procedimientos de continua formación y deformación de lo orgánico, con la idea de involución creadora que remite directamente a la obra quizás más radical del pensamiento de Bergson, L'Évolution créatrice (2007).

Deleuze en Mille Plateaux (1980) escribe a modo de obertura lo que expresa un cuerpo sin órganos:

De todas maneras tienes uno (o varios), no tanto porque preexista o venga dado hecho — aunque en cierto sentido preexiste-, sino porque de todas maneras haces uno, no puedes desear sin hacer uno - te espera, es un ejercicio (exercise), una experimentación (expérimentation) inevitable, ya hecha en el momento en que la emprendes, no hecha en tanto que no la emprendas. No es tranquilizador, puesto que puedes fallarlo. O bien puede ser terrorífico, conducirte a la muerte. Es no-deseo tanto como deseo. De ningún modo es una noción, un concepto, más bien es una práctica, un conjunto de prácticas (un ensemble de practiques). (p. 185-186).

Exhortación en el tono: desde el inicio el texto se expresa con este tono apelativo que remite al modo de un consejo de lo que pesa ahí en un cuerpo sin órganos. Nos recuerda ciertamente a la tradición de la filosofía helenística, quizá epicúrea o estoica, que entendían la actividad filosófica como una 
especie de repetición de ciertas prácticas, de un cierto cultivo de sí en vistas de un apostado bienestar del ánimo. Una serie de prácticas, por cierto que sí; felicidad, en apariencia, no, si ajustamos nuestra lectura a la de serie de ejemplos desarrollados a través del primer tramo de texto. En el CsO está implicado, creemos, un cierto entendimiento de la tentativa, o al menos de la experiencia de su despliegue, tentativa que explica hasta cierto punto la naturaleza práctica y no meramente conceptual de su enunciación. Los ejemplos visitados por Deleuze en este primer segmento de texto dan cuenta de las notas esenciales de este o estos procesos de constitución y desintegración progresivos que toman por asalto a un cuerpo: el hipocondríaco, el paranoico, el esquizofrénico, el drogadicto, el masoquista dibujan la escena de un teatro lo suficientemente macabro de lo que en un principio se podría pensar acerca del programa de formación de un $\mathrm{CsO}$. La realidad de la falla y la muerte parece siempre acechar a dicho proceso, es por esto que es necesario para constituir un CsO un arte efectivo de la prudencia, una arte de la tentativa, de un esbozo diagramático y de la dosis que permita definir las secuencias de experimentación, el despliegue de una serie de prácticas que inquieren por deshacer la idea de organización que define los rasgos de toda significación, subjetivación y organismo posibles. Deshacerse a fin de cuentas de toda trascendencia organizada que impone formas, funciones y jerarquizaciones para sacar de ello un provecho, un producto conforme a la norma de una utilidad. Inversamente, si el CsO no es extensivo a una finalidad, tampoco posee un origen ni un fundamento determinable, siempre se está en medio de su proceso, en el intervalo que da cuenta de su constante producción, en fin, de su configuración en devenir. Es por esto que la pregunta qué es un CsO, una pregunta por la esencia o el fundamento no cobra sentido aquí, ya que no hay nada esencial, estable y organizado en su expresión e implicaciones. Lo que importa al CsO no son individuos, sedimentaciones o concreciones, i.e., todo lo que define para Deleuze los estratos; sino más bien lo que interesa en este proceso es captar flujos, fluctuaciones, oscilaciones, recorridos y desviaciones: toda una metafísica de la potencia que intenta expresar el devenir.

Volvamos al inicio, repitiendo así el título del texto: ¿Cómo hacerse un cuerpo sin órganos? Quizá una vía plausible de análisis pueda ser ensayada a partir de la observación de la estructura de la pregunta que inaugura su formulación.

En primer lugar, la posibilidad efectiva del CsO en cuanto proceso se nos presenta de entrada como una interrogación, como una especie de campo de alternativas que provoca a pensar un desarrollo de constitución de algo que no nos es dado, al menos inmediatamente, a la experiencia en general. El Cómo de la pregunta nos reenvía directamente a la necesidad de regulación 
de un proceso, no se interroga por la substancia de la cosa, sino por su proceso de configuración. La realidad del $\mathrm{CsO}$ parece expresarse bajo una premisa esencial: es necesario dar cuenta de las instancias decisivas de un proceso de fabricación o de constitución de aquel cuerpo, de modo que nos sea accesible su operación. Bajo qué pasos, bajo qué transformaciones y recursos es posible hacer un $\mathrm{CsO}$, pareciese ser lo imperativo que presenta desde el inicio el estilo de planteamiento de Deleuze. Si la verdad del CsO es, como consecuencia, la verdad de su propio proceso, este proceso habría de construir la realidad y, al menos esbozar, las condiciones de esta singular forma de pensar una existencia. Debe señalar, pues, una dirección o un sentido por donde debe pasar la realidad devenida de un $\mathrm{CsO}{ }^{3}$

Esa es la dirección que define y señala a ese Hacerse, un verbo en infinitivo, una especie de acontecimiento puro de la producción de ser, pero de un ser del devenir, o del puro devenir sin ser, como ya había sido pensado por Deleuze en Logique du sens (1969). Recordemos: "El acontecimiento no es lo que sucede (accidente); está en lo que sucede el puro expresado que nos hace señas y nos espera" (p. 175). Esos gestos que definen el acontecimiento se vuelven a repetir para la enunciación del CsO, una forma específica de expresión que da razón al tono apelativo que constantemente es usado por Deleuze para dar cuenta de los segmentos que definen este proceso de formación. Primero, no es posible representar el CsO como un mero accidente, como algo que acaece o se dice del cuerpo, de una sustancia. Es, en efecto y en segundo lugar, la potencia de conexión y de relaciones lo que define su operatoria. Es por esta razón, a nuestro juicio, que Deleuze vincula ese hacerse del CsO con el libro de la Ética de Spinoza, ya que para poder hacerse un $\mathrm{CsO}$ es necesario determinar al cuerpo en sus relaciones, en la potencia de conjugar y conectar cada vez sus propios elementos en proceso, sin tener en vista la regulación de un fin o la individuación idéntica a sí misma en una determinada entidad particular. Deleuze insiste en ello: "Conectar, conjugar, continuar (Connecter, conjuguer, continuer)" son los verbos que definen, por diagrama (diagramme) en oposición a todo programa funcional (programme), la operación del CsO: "Solo ahí el CsO se revela como lo que es, conexión de deseos, conjunción de flujos, continuum de intensidades" (DELEUZE, 1980, p. 199). Es por esto que, negativamente, se puede fallar en el proceso de formación de un CsO e incluso morir en la

\footnotetext{
${ }^{3}$ Del mismo modo que la literatura, como la existencia y la vida misma, el CsO es una cuestión que se juega en el devenir. En otras palabras, la apuesta metafísica delezeana, del mismo modo como la que se deja leer en el pensamiento de Bergson, siempre se tratará de dar cuenta del devenir en cuanto tal, sin la necesidad de establecer un soporte o sustancia preexistente que de lugar al acontecimiento de su cambio, y de sus consecuentes aunque eventuales transformaciones. Volveremos sobre ello. E texto que nos parece decisivo para confirmar esta sugerencia, que por cierto excede los límites de lo que intentamos revisar en este lugar, no sería otro que "Devenir-intense, devenir-animal, devenirimperceptible" (DELEUZE, 1980, p. 284ss.)
} 
instalación de su proceso: el hipocondríaco, el paranoico, el esquizofrénico, el drogadicto, el masoquista al deshacer el organismo de modo imprudente, a violentos martillazos, quedan en la clausura y en el vaciamiento de sus propios órganos, ya que nada pasa, nada se conecta o se conjuga con ellos; el todo de la materia fluctuante que expresa de mejor manera un $\mathrm{CsO}$ queda así bloqueado, sedimentado por las estratificaciones que definen funcionalmente lo orgánico: "Sus enemigos no son los órganos. El enemigo es el organismo. El CsO no se opone a los órganos, sino a esa organización de los órganos que llamamos organismo" (DELEUZE, 1988, p. 196) concluye contundentemente Deleuze.

Esto nos da una seña más acerca de lo que se enuncia con el CsO: la apuesta de un $\mathrm{CsO}$ es dar expresión a la realidad de los variables procesos de formación ya no de individuos sino que de singularidades. Por esto sostenemos que es convenientemente una fórmula en dos sentidos complementarios que definen dicha operación: primero, por ser la reserva de un mínimo de forma que pueda captar los flujos, enlaces y transformaciones que cada vez van componiendo su realidad y; en un segundo sentido, porque ese mínimo formal funciona como el operador mismo del proceso de esas transformaciones que implica la constante redistribución de aquellos flujos elementales que lo configuran. En consecuencia, la categoría ontológica que conviene como forma de determinación específica para un $\mathrm{CsO}$, no puede ser otra que la de relación, potencia de relación, una potencia de afectar y de ser afectado. A cada relación de movimiento y reposo que expresa la realidad de un CsO corresponde un grado de potencia, un tipo de intensidad que la afecta, lo cual permite ligar esta operación con Spinoza en el texto de Deleuze. De este modo, nada sabremos del cuerpo hasta que sepamos lo que un cuerpo puede, cuáles son sus afectos, cómo puede o no componerse con otros afectos, en una palabra, cuánto es su coeficiente potencial de devenir. Es por esto que solo lo que involuciona evoluciona, ya que la potencia de transformación no se entiende desde la adición de partes sobre partes para formar una suma total cerrada sobre sí misma; sino al contrario, el hacerse de un $\mathrm{CsO}$ requiere de un primer deshacerse de la organización de los órganos para así transformarse, lograr la toma de consistencia de sus relaciones en variación continua, i.e., dar paso a los flujos que cada vez lo componen y descomponen en un campo abierto de relaciones no totalizables en una suma: ahora entendemos la naturaleza de esos verbos antes citados, potencia de conexión de una multiplicidad de deseos, potencia de conjunción de fluctuaciones, potencia de traspaso de intensidades. Potencia que no es sino, una potencia de lucha frente a las formas de determinación de lo trascendente de lo Uno, lo Mismo y lo Extenso.

O bien, leyendo el final de la pregunta, un cuerpo sin órganos significa algo más que un término nominal, es la excedencia del proceso como término, 
lo que es enfatizado por Deleuze, radicalizando su enunciado gracias a la teoría del discurso de Guillaume en el uso del indefinido Un. La operación gramatical de este artículo señala la radical despersonalización, desindividuación del proceso que es coronado por el CsO, dando paso así a un proceso infinito de singularización y no ya de generalización y determinación como sería el caso del uso lingüístico de uno definido, el artículo El ${ }^{4} \mathrm{El}$ espacio que abre un $\mathrm{CsO}$, quizá al modo de la noción de contemplaciones en Plotino, articula de modo diverso y variable un abierto colectivo de: "elementos, cosas, vegetales, animales, herramientas, hombres, potencias, fragmentos de todo eso; pues no puede hablarse de "mi" cuerpo sin órganos, sino de "yo" en él, lo que queda de mí, inalterable y cambiando de forma, franqueando umbrales" (p. 200). Un CsO, bajo esta óptica, ya no puede ser interpretado como un sujeto, sujeto de o al enunciado, ya que cada vez se encuentra frente a la necesidad de sustraerse, reducir su forma, a toda posible organización de significaciones en vistas de la determinación de un sentido que pareciese preexistir. De este modo, hacerse un CsO se expresa con la operación de sustracción de toda trascendencia significante, subjetiva y orgánica con que habitualmente son pensados los fenómenos de cambio y alteración que se efectúan conforme a un fin trascendente. Un CsO constituye así lo que Deleuze llama campo de inmanencia o plan de consistencia que se halla siempre en oscilación con todos los estratos, toda trascendencia que inquiere por determinarlo, i.e., dar forma a un cuerpo como función de lo orgánico: "El campo de inmanencia no es interior al yo, pero tampoco procede de un yo exterior o de un no-yo. Más bien es como el Afuera absoluto (le Dehors absolu) que ya no conoce los Yo, puesto que lo interior y lo exterior forman igualmente parte de la inmanencia en la que se han fundido" (DELEUZE, 1980, p. 194).

\footnotetext{
${ }^{4}$ La influencia de la lingüística de Guillaume, como también la de Hjelmslev, es profunda en el pensamiento de Deleuze. En ambas teorías, tomadas desde su generalidad, intentan dar razón del cambio lingüístico no a través de variables externas que determinarían un cierto devenir de las formas de uso que puede tomar un determinado sistema de lengua, desde el punto de vista de su norma de uso. Para ambas teoría el cambio no se suscribe tanto por el decaimiento de un determinado uso dado por la presencia o desaparición de vocablos o materiales lexicales históricamente dados, sino más bien los procesos de variación penden de la transformación sintáctica que determinadas partículas afectan a la materia de sentido que un enunciado pone en movimiento en su instancia de emisión. Para Guillaume, este mínimo artículo 'un', su presencia o su alternancia con el uso de 'el', provoca una divergencia absoluta de los efectos de sentido que un enunciado puede tomar, dirigiéndose a un proceso de singularización en el primer caso, o bien, de generalización en el segundo. Por cierto, para Deleuze, el primer caso posee relevancia en la medida en que el devenir no opera sobre segmentación y por una generalización de individuos en vista de una totalización de un conjunto significante, sino todo lo contrario, la operación del artículo indefinido señala o expresa la apertura del devenir que afirma la multiplicidad de singulares expresables por una línea enunciativa que puede variar libremente en sus recorridos. Para el problema del enunciado en este contexto, remitimos a "Postulats de la linguistique" (DELEUZE, 1980, p. 95ss.); para el estatuto del artículo definido/indefinido, Cf. GUILLAUME, 1964, p. 143ss. y 157ss. Sobre este problema referido al estatuto general del lenguaje en el campo de una teoría del estilo, ver nuestro artículo "Ensayo sobre estilo y variación" (2006).
} 
En efecto, la diversas modalidades del proceso infinito de producción de un CsO señalan la destrucción de la regla que da lugar al producto, convirtiendo al producto en un punto de indeterminación radical que inquiere por verse nuevamente determinado. Hacerse significa, entonces, esencialmente deshacerse de los órganos, del organismo que siempre tiene un fin en vistas en cada proceso de acción. La acción entonces adquiere este rasgo involutivo, el cual opera en ese $S e$ dirigido hacia una transformación, despersonalizando, desindividualizando y desubjetivando, a fin de cuentas, sustrayendo las condiciones de la experiencia en general de lo posible, para así ganar una inmanencia que cada vez requiere de verse sustraída de toda trascendencia determinante. Reservar un mínimo de forma, finalmente, para poder así transformarla bajo un criterio prudente de la tentativa que debe acomodarse cada vez en la trascendencia que busca por definir, determinar y ubicar su propio emplazamiento, ya que:

\begin{abstract}
Hace falta conservar una buena parte del organismo para que cada mañana pueda volver a formarse; también hay que conservar pequeñas provisiones de significancia y de interpretación, incluso para oponerlas a su propio sistema cuando las circunstancias lo exigen, cuando las cosas, las personas, e incluso las situaciones, te fuercen a ello; y también hay que conservar pequeñas dosis de subjetividad, justo las suficientes para poder responder a la realidad dominante. Mima los estratos. No se puede alcanzar un $\mathrm{CsO}$, y su plan de consistencia, desestratificando salvajemente. Por eso encontrábamos desde el principio la paradoja de esos cuerpos lúgubres y vaciados: se habían vaciado de sus órganos en lugar de buscar los puntos en los que podían paciente y momentáneamente deshacer (défaire) esa organización de los órganos que llamamos organismo. (DELEUZE, 1980, p. 199).
\end{abstract}

Sustraerse de la forma: el CsO no puede ser sino la operación o la fórmula que se enfrenta cada vez a la organización de los órganos. Reservar o sustraerse a un mínimo de forma es involucionar, llevar al mínimo la organización para así causar un aumento de potencia de conexión, un aumento del potencial de transformación para dar lugar a la emergencia de nuevas formaciones: en suma, una involución creadora que no puede preveer aquello que se da en su proceso de configuración, el CsO es entonces la expresión conveniente de un proceso abierto a una novedad absoluta de sus propias conexiones. En una palabra, una duración abierta a otras duraciones, como ya lo insinuaba Bergson. 
Expresiones del movimiento vital, afectos que intensifican los grados de germinación que cada vez se actualizan en un campo general de indeterminación pueden resumir la operación prudente convocada bajo la expresión de CsO. Deleuze por medio del CsO define los rasgos de una etología de la sensación, afectos como lo referirá después, que componen cada vez a un cuerpo. Se sustrae así, por medio de Spinoza, de una metafísica de la sustancia, para configurar los marcos de un empirismo trascendental o un escepticismo frente a lo trascendente o una metafísica de la potencia que afirma la singularidad y la contingencia; en fin, entender el cuerpo como los modos y expresiones del ser siempre deviniendo: "se definirá a un animal o a un hombre no por su forma, ni por sus órganos y funciones, ni tampoco como un sujeto; se lo definirá por los afectos de los que es capaz" (DELEUZE, 1988, p. 124). Cuando hemos señalado que este proceso toma lugar bajo la expresión involución creadora, en el fondo no hallamos sino la apuesta por un vitalismo radical que parece coincidir con la teoría de la experiencia que impone el proceso y la enunciación del CsO. Esta teoría de la experiencia deviene en un arte de la experimentación cuando en cada prudente tentativa deshacía lo que orgánicamente aparecía garantizado por las formas de determinación de lo trascendente, los estratos en el vocabulario de Deleuze. En efecto, lo orgánico se reconoce por los caracteres que se disponen de modo definido y cuantificable en una extensión: la tarea de un $\mathrm{CsO}$ era expresar las relaciones intensivas con que puede ser producido un cuerpo como un campo abierto de fuerzas siempre en constante cualificación. Esta captación de fuerzas implica una novedad respecto de un posible análisis del $\mathrm{CsO}$, a saber, que la determinación intensiva, esa diferencia intensiva que nuevamente hace actual la operación del un hacia cada órgano, sienta las bases para pensar un vitalismo esencial que recorre y haría plausible el proyecto del CsO. Producir las condiciones para pensar un campo de inmanencia o campo trascendental sin sujeto ni objeto, habitado por singularidades puras en devenir, será lo que Deleuze (2003), finalmente, sellará simplemente en la expresión una vida: "una vida inmanente que es pura potencia (pure puissance), e incluso beatitud a través de los sufrimientos y las debilidades" (p. 362).

Desde una simple lectura al texto del CsO reconocemos no solo múltiples sino heterogéneas referencias que intentan dar una imagen de esta operación o fórmula: los ejemplos tomados de la psicología clínica, la literatura de Artaud, Lawrence o Castaneda, la filosofía de Spinoza, pero también soterradamente la de Nietzsche y Plotino, la filosofía oriental, el amor cortés, la lógica medieval al lado de retazos de fenomenología, no parecieran sino estar en función de desmembrar, i.e. desorganizar para dar otra fisonomía, a aquel otro cuerpo que habitualmente reconocemos como 
nuestra propia tradición de pensamiento. En lo que sigue, nos proponemos insistir solo en una de todas estas, quizá la más implícita de estas, en la de Bergson, con el fin de esbozar algunas líneas que nos ofrezcan un paisaje de la conexión entre estas dos formas de actividad filosófica en el horizonte de una teoría general de la vida.

Para Bergson en L'Évolution créatrice (2007) la noción de vida se presenta en un despliegue de "elementos que no gozan de existencia real y separada. Lo que hay son vistas múltiples del espíritu sobre un proceso indivisible. De ahí la contingencia radical en el progreso, inconmensurabilidad entre lo que precede y lo que sigue, en una palabra: duración" (p. 57). Contingencia e inconmensurabilidad, esto es, flujos, continuidades, duraciones que expresadas por las palabras de Bergson guardan total convergencia con las de Deleuze cuando este refiere al proceso de formulación de un CsO. A su vez, esos rasgos y articulaciones, que conformarán la tesis fuerte del élan vital como una especie de traducción ontológica del concepto de duración, se hallan convocados en la enunciación del CsO que intenta expresar o dar una imagen de lo múltiple del devenir. Contingencia e imprevisión, inconmensurabilidad entre los estados de un proceso, confluyen en un abierto vitalismo que anima la noción de CsO tanto como la evolución y transformación de los entes vivientes en el pensamiento maduro de Bergson. La filosofía de Deleuze (2003), en este sentido, se nos presenta como una extensión del proyecto bergsoniano, lo cual: "no significa solamente una admiración renovada por un gran filósofo, sino una respuesta o una prolongación de su tentativa" (p. 313), i.e. no un comentario o interpretación, sino más bien la repetición del gesto original que da lugar a un proyecto en materia filosófica. El CsO desde esta perspectiva no es una simple lectura, aunque esta esté implicada atentamente, sino una transformación de la noción del élan vital, en la medida en que lo que está en juego es la radicalización de las tesis de resistencia frente a lo trascendente y de la intensidad de las relaciones y las fluctuaciones que ponen en figura a cada una de sus determinaciones conforme a un entendimiento de lo real.

Damos aquí un esbozo de esta tentativa de comprensión de uno y otro pensamiento. Tanto el CsO como el élan vital cumplen el rol de sustraerse del influjo de la trascendencia: tanto el mecanicismo de la causalidad exterior como de la teleología de la naturaleza que compone las partes en virtud de un todo hipotético son puestas en entredicho por ambas operaciones. Los capítulos primero y segundo de L'Évolution créatrice responden a estas dos formas de explicación de la germinación y desarrollo de los organismos (Cf. BERGSON, 2007, p. 88ss). La disociación y el desdoblamiento de la vida en cada una de las especies es otro rasgo que rescata Deleuze del élan vital. Sabemos que la divergencia que opera en la transformación de la serie animal 
en Bergson (2007) se expresa bajo la razón de que "la vida es, fundamentalmente, tendencia a actuar sobre la materia bruta" (p. 97). Esto no es sino el rasgo esencial que caracteriza la imprevisibilidad de las acciones $y$, fundamentalmente, las elecciones que cada especie esboza en esta constante lucha con la materia que siempre se plantea como un obstáculo a su desarrollo. De modo semejante, el CsO debe en cada instancia guardar cierta dosis del juicio de lo trascendente para sustraerse de la organización, de la interpretación significante y de la subjetividad, como un momento estratégico para provocar la involución. Sin embargo, hemos visto que la involución se dice de la forma que toma cada vez una porción de materia, de elementos en constante distribución. Aquí podríamos observar una diferencia de fondo con la idea de materia, y que explica ese ánimo de réplica y prolongación que el discurso filosófico de Deleuze imprime en sus comentarios a algunos filósofos de la tradición. Y este punto específico se explica en virtud de que para Deleuze (1980) a diferencia de Bergson:

Materia es igual a energía. Producción de lo real como magnitud intensiva a partir de cero. Por eso nosotros tratamos el CsO como el huevo lleno anterior a la extensión del organismo y a la organización de los órganos, anterior a la formación de los estratos, el huevo intenso que se define por ejes y vectores, gradientes y umbrales, tendencias dinámicas con mutación de energía, movimientos cinemáticos con desplazamiento de grupos, migraciones, y todo ello independientemente de formas accesorias, puesto que los órganos sólo aparecen y funcionan aquí como intensidades puras. (p. 159).

Producción de intensidades, y no de exteriorizaciones en el campo de la materia: el caso de Bergson considera que tanto el proceso de constitución como el desarrollo de una especie, aunque con fines imprecisos e imprevisibles, se da específicamente por la selección entre intensidades virtuales con que se presenta la vida, selección que, eliminando una sección de ese haz virtual, explota por un élan vital que inquiere por verse actualizado en la exterioridad material de la naturaleza. Una duración que se abre para intervenir otras duraciones, proceso que se identifica con la idea de evolución que rompe el principio adaptativo, tanto mecánico como dinámico, haciéndola devenir creadora, a saber, establecer la novedad que por exteriorización se presenta como un principio de indeterminación. Y así lo confirma Bergson (2007):

El papel de la vida es insertar indeterminación en la materia. Las formas que va creando al compás de su evolución son indeterminadas, es decir, que no podían preverse; así también la 
actividad de la cual esas formas debían ser vehículo ha ido siendo indeterminada, quiero decir, más libre. (p. 127).

$\mathrm{El} \mathrm{CsO}$, en cuanto lectura al impulso vital de Bergson, no indica sino que la estabilidad de un cuerpo reside en su inestabilidad -nunca cesa de cambiar cualitativamente (alloiosis). ${ }^{5}$ Ahora bien, un cuerpo, concebido como un sistema relativamente cerrado, es posible de derecho aislar dentro de la continuidad de la materia. Lo que es real de un cuerpo son dos cosas: la continuidad moviente en el todo y el continuo cambio de forma en el interior del cuerpo viviente con que toda vez se entrecruza formando su propio campo. Es necesario destacar que por forma se entiende aquí solo esta especie de instantánea dentro de un proceso. Lo que nuestra percepción en general hace es hacer sólido el flujo continuo de lo real o de la totalidad abierta en imágenes discontinuas y discretas en vista de un análisis posible. Esto ocurre bajo la condición de la necesaria evolución y adaptación a un determinado entorno de acción. Todo cambio toma lugar en el todo, sin embargo, estos son recibidos por el sistema de percepción del viviente solo en superficie. Un sistema como el nuestro, con sus evolucionados hábitos de representación, dejan de lado del movimiento de la vida o ponen simplemente su interés en la parte inmóvil y en un determinado plan de movimiento más que en el movimiento mismo: esto es, sustraído de la duración del proceso. Todos los tipos de actos son reducidos a la imagen de un movimiento simple o un movimiento en general, y el conocimiento viene a instalarse en un estado de cosas estable más que en su cambio constante. Brevemente, es posible decir que desarrollamos tres tipos de representaciones que se corresponden a tres categorías de palabras: cualidades (adjetivos), formas de esencias (sustantivos) y actos (verbos). Mientras que los dos primeros están diseñados para capturar estados, el último está en relación con el movimiento y expresa

\footnotetext{
${ }^{5}$ La categoría de alteración (alloiosis) que acá señalamos para intentar explicar el fondo metafísico es, con toda seguridad, la de más difícil comprensión en el contexto de Aristóteles en la justa medida en que, como lo recordamos desde Física en el inicio de nuestra discusión, siempre se halla enlazada con la realidad activa de la sensibilidad. Pero es por esta razón, que asumimos como oscura, creemos puede explicar de modo específico lo que se plantea de fondo tanto por élan vital bergsoniano como por el CsO delezeano. Citamos, nuevamente, desde Física: "La alteración es la actualidad de lo alterable en tanto que alterable, o en términos más conocidos, es la actualidad de lo potencialmente activo y lo potencialmente pasivo en cuanto tales, tanto en general como en cada caso particular (por ejemplo, en los procesos de construir y curar)" (202b25). En efecto, la alloiosis siempre presentará ese valor diferencial en la medida en que su potencial actualidad señala el proceso siempre material de construcción o destrucción; de enfermedad y de salud, sin la necesidad de que sus extremos sean pensables como previamente preexistentes: esto no es sino el elemento esencialmente diferencial que define la vida, de sus explosiones imprevistas (élan vital) o de sus trasformaciones intensivas (CsO), ya que "no hay entonces ni identidad ni igualdad ni semejanza en las alteraciones, ya que la diferencia en las cualidades hace que las alteraciones sean específicamente diferentes, ni la alteración es única, como tampoco serán únicos los movimientos locales" (249b10).
} 
algo que podríamos estimar como difícil de pensar (Cf. ANSELL-PEARSON, 2002, p. 50): la vida de un cuerpo, para nosotros, qua virtual o infinitivo o acontecimiento o campo trascendental o plano de inmanencia ${ }^{6}$, debe toda vez sustraerse de las formas de la trascendencia, cuya operación es el modelo del $\mathrm{CsO}$, de toda norma determinación que lo clausura por definición. O bien, un $\mathrm{CsO}$ que radicaliza las instancias durables de un proceso llevándolas a un mínimo de forma capaz de captar las fuerzas que cada vez lo componen: esto es de modo definitorio una involución. ${ }^{7}$

Este cambio de dirección, involución ${ }^{8}$ por evolución, implica a nuestro juicio una separación radical con el proyecto de Bergson en la medida en que para Deleuze el CsO proyecta un devenir inhumano en el agenciamiento

\footnotetext{
${ }^{6}$ En esto seguimos a Prado quien, a nuestro juicio, coliga de modo más eficiente el juego de conceptos que aquí hemos abordado a partir del CsO: nociones como campo, plano, la figura del huevo, y finalmente, diagrama, no podrían ser sino expresiones que replican la forma de conceptualización tal propia de la fenomenología y su característica trascendental (Cf. PRADO, 1998, p. 308-309). Citamos para esto a Prado (1998): "Añadamos que ese campo -el lugar donde se producen y circulan los conceptos- no es pensable por sí mismo. Su definición y el dibujo de su cartografía solo son posibles por la definición simultánea de los conceptos que lo pueblan o lo llenan. Si los conceptos tienen necesidad de un campo virtual pre-existente, el plano no puede subsistir sin los conceptos que lo frecuentan y yerran como tribus nómadas en el desierto; o aún, que lo marcan como las islas hacen archipiélago en el océano. Pero que la metáfora no nos engañe: hay desiertos que no son habitados y la superficie del océano no siempre esta interrumpida o 'punteado' (pointillée) por islas. Así, una vez más, si no hay concepto sin plano, no hay plano sin conceptos que inscriban, en este 'elemento' fluido y virtual, las superficies y los volúmenes que lo marcan como las series de acontecimientos, recubriéndolo como las baldosas innumerables, distendiendo así ese medio indivisible" (p. 307). A este recorrido, cartografiable, a ese errar nómade por desiertos, a la configuración, digamos, incoativa de cada ensayo y esbozo, viene a nuestra óptica a responder la realidad operacional, pero profundamente transformacional, que hasta aquí hemos indicado como un cuerpo sin órganos.

${ }^{7}$ Pero involución no es destrucción de los órganos, en razón a lo que enfatizábamos desde Guillaume: del mismo modo que ha de haber un $\mathrm{CsO}$, se debe evitar a toda costa a ese otro gran peligro que, por proliferación cuasi cancerosa, expresan los OsC (órganos sin cuerpo), cuestión que ulteriormente con la asistencia de Lacan, explora Zizek (2004). A Deleuze lo que le interesa es dar con el punto que por generación espermática libera a las formas, afirmando su carácter metamórfico, cuasi ovidiano, de un CsO: ''Un' vientre, 'un' ojo, 'una' boca: el artículo indefinido no carece de nada, no es indeterminado o indiferenciado, sino que expresa la pura determinación de intensidad, la diferencia intensiva. El artículo indefinido es el conductor del deseo. No se trata de modo alguno de un cuerpo desmembrado, fragmentado o de órganos sin cuerpo (OsC). El CsO es justo lo contrario. No hay en modo alguno órganos desmembrados con relación a una unidad perdida, ni retorno a lo indiferenciado respecto de una totalidad diferenciable. Hay distribución de razones intensivas de órganos, con sus artículos positivos indefinidos, en el seno de un colectivo o de una multiplicidad, en un agenciamiento, y según conexiones maquínicas que actúan en un CsO. Logos spermaticos" (DELEUZE, 1980, p. 203). Para la discusión de Zizek sobre este punto y su revisión crítica que aceptamos, ver el artículo "Demanding Deleuze" de Ansell Pearson (2004, p. 37-38, específicamente).

${ }^{8}$ Fue relevante para este ensayo el trabajo de Ansell-Pearson (1999, p. 139ss), quien realiza una extensa y detallada inspección sobre los caracteres de esta diferencia, la cual se cifra en su especificidad en el entendimiento del proyecto de una etología y la diversidad de fuentes y desarrollos que componen esta disciplina parte de la biología. La heterogeneidad de las fuentes de Bergson y Deleuze en este aspecto marca, al menos desde un análisis textual, una excelente evidencia para estas distancias, aunque a nuestro juicio, esta diferencia es mucho más radical e involucra una réplica y a la vez una prolongación de ambos proyectos metafísicos: "La hipótesis científica y la tesis metafísica no cesan de combinarse
} 
propio de ese proceso. Y esto es así, ya que Bergson (2007) manifestará, finalmente, este ánimo por la trascendencia en los marcos del trabajo filosófico, que no es más en este contexto, que pensar lo humano:

La filosofía no puede ser más que un esfuerzo para fundirse de nuevo en el todo. La inteligencia, reabsorbiéndose en su principio, revivirá en sentido inverso su propia génesis. Empresa que no podrá conseguirse de golpe: será necesariamente colectiva y progresiva. Esta consistirá en un intercambio de impresiones que, corrigiéndose mutuamente y superponiéndose unas a otras, acaben por expandir en nosotros la humanidad y por obtener que ella se trascienda a sí misma. (p. 193).

Insistimos este cambio de dirección es plenamente una nota spinoziana, también nietzscheana, que aleja el programa del $\mathrm{CsO}$ del élan vital bergsoniano: "no saben de antemano de lo que son capaces en lo bueno y en lo malo, no saben de antemano lo que puede un cuerpo o un alma en tal encuentro, en tal dispositivo, en tal combinación" (DELEUZE, 1988, p. 125). Esto no es sino una posible comprensión de lo que se deja leer en Spinoza (2007), en su Ética, en la Proposición XIII; damos cita al escolio de esta proposición para dar cuenta de nuestro punto:

\begin{abstract}
Con todo, diré en general que, cuanto más apto es un cuerpo que los demás para obrar o padecer muchas cosas a la vez, tanto más apta es su alma que las demás para percibir muchas cosas a la vez; y que cuanto más dependen las acciones de un cuerpo de ese solo cuerpo, y cuanto menos cooperan otros cuerpos con él en la acción, tanto más apta es su alma para entender distintamente. (p. 129).
\end{abstract}

Lo que parece importar finalmente a Deleuze, retomando a Spinoza, es enfatizar que la realidad del cuerpo es una realidad compuesta, pero compuesta de fuerzas que acrecientan o menguan su potencia, y su potencia se dice en todo momento de una potencia abierta y amplia de conexión con otros cuerpos: el cuerpo sin órganos, deshaciendo el estatuto funcional que lo define en cuanto organismo, debe ser la expresión de este constante deshacerse de funciones en vista siempre de la configuración de una campo abierto de conexiones que cada vez afirman su propia potencia, i.e., una inmanencia absoluta que se actualiza en la realidad de un CSO.

en Bergson con el fin de dar imagen y perseguir (retracer) una experiencia integral" (DELEUZE, 2003, p. 315). Y en respuesta a esa experiencia integral, su inscripción, es que se hace necesario un devenir de los propios conceptos que genera en esta ocasión un $\mathrm{CsO}$, al sustraerse toda vez de una típica categorial que domine y asegure sus formas, correctas y precisas, de presentación verdadera. 
En este sentido podemos decir, en suma, que el CsO no es más que la expresión de la potencia de afecto en las condiciones de la experiencia real, la cual siempre debe conservar un mínimo de forma, ser en términos efectivos una fórmula. La fórmula, en definitiva, es un potencial de transformación que no requiere de una separación con la materia, sino hacerla y tomarla como intensa y fluctuante, justo lo suficiente para poder decir que se ha puesto en crisis su actual organización que involuciona o se inclina hacia otras, nuevas, formas de composición.

RUIZ STULL, M. BwO's Formula. A Bergsonian approach to utterance's conditions. Trans/ Form/Ação, (Marília); v.34, n.1, 2011, p.131-148.

ABSTRACT: Taken from Artaud by Deleuze and from the writing of Logic of Sense (1969), the expression of a body without organs (BwO) continues to cause at least perplexity. In his statement plots crucial points in the philosophy of Deleuze from his theory of the event and the difference, to an analytical definition and the desire, to a certain concept of life that would articulate the process of their conception. Without underestimating the above and the profuse use and current measurements that has claimed this, our aim is to link the BwO with the conception of life, which according to our reading, is at the source of their formulation. This remains the underlying text, and our proposa settle on to determine the degree of influence that has the vitalism of Bergson in the design of this formula: From this point of view is plausible that the CSO is an operation that is based on crucially on a careful reading of the bergsonian élan vital. In brief, if the $\mathrm{BwO}$ is an involution of their creative process by definition, is merely because their start-up means and takes place in a constant struggle and radical increasingly involves the subtraction or dismantling of the idea of organism.

KEYWORDS: G Deleuze. H Bergson. Life. Organism. BwO.

\section{Referencias}

ALLIEZ, Eric. La condición CsO, o de la política de la sensación. Revista Laguna, Tenerife, v. 15, p. 91-106, 2004.

ANSELL-PEARSON, Keith. Germinal life. New York: Routledge, 1999.

Demanding Deleuze. Radical Philosophy, London, n. 126, p. 33-38, 2004.

ARISTÓTELES. Física. Madrid: Gredos, 2000.

BERGSON, Henry. L'Évolution créatrice. Édition critique de F. Worms. Paris: PUF, 2007.

DELEUZE, Gilles. Différence et répétition. Paris: PUF, 1968.

Logique du sens. Paris: Les Éditions de Minuit, 1969.

Mille Plateaux. Paris: Les Éditions de Minuit, 1980. 
DELEUZE, Gilles. Spinoza: practical philosophy. San Francisco: City Lights Books, 1988.

Deux régimes de fous. Paris: Les Éditions de Minuit, 2003.

GUILLAUME, Gustave. Langage et science du langage. Paris: Nizet, 1964.

PRADO, Bento. Sur le 'plan de immanence'. In: ALLIEZ, Eric. Gilles Deleuze, une vie philosophique. Luisant: Synthélabo, 1998. p. 305-23.

RUIZ STULL, Miguel. Ensayo sobre estilo y variación. Onomázein, Santiago, v. 14, n. 2, p. 171-196, 2006.

SPINOZA, Baruch. Ética. Madrid: Alianza, 2007.

ZIZEK, Slavoj. Organs without bodies: on Deleuze and consequences. New York: Routledge, 2004 
